\title{
Methodology Development at NYULMC Microscopy Core - Correlative Light and Electron Microscopy Applications
}

\author{
Alice F. Liang ${ }^{1,2,{ }^{*}}$, Chris Petzold ${ }^{1}$, Kristen Dancel-Manning ${ }^{1}$, Yan Deng ${ }^{1}$ and Michael Cammer ${ }^{1}$ \\ ${ }^{1 .}$ OCS Microscopy Core, New York University Langone Medical Center, New York, USA \\ 2. Department of Cell Biology, New York University Langone Medical Center, New York, USA \\ * Corresponding author
}

The Microscopy Core at New York University Langone Medical Center (NYULMC) established in 2005 is based on electron microscopy services. Light microscopy services were implemented in 2009 and the Core became a fully functional Microscopy Core.

Similar to most Microscopy Cores, our light microscopy (LM) services focus on training for the correct use of microscopes, and keeping all the microscopes in a nice working state. Image analysis is another major LM core service, which helps users to better understand their imaging data. Our electron microscopy (EM) services are very broad, ranging from all different kinds of sample preparation to image acquisition. Given the fact of rapid development of light and electron microscopes, catching up with new technology is one of the key points for a modern Microscopy Core. The advantage of combining light and electron microscopy services in a single core provides a unique position for the Microscopy Core to carry projects over from light microscopy to electron microscopy. So, correlative light and electron microscopy (CLEM) becomes a hot selling point for our Microscopy Core services.

Fluorescence microscopy has opened the way to study protein localization, interaction, and function in live cells. A huge effort has been put in this field during the last 20 years to improve the resolution of light microscopes, and super-resolution microscopes are good examples to localize protein at single molecule levels. However, precise localization of protein at high resolution and at the ultrastructural level can only be studied by electron microscopy (EM). By combining light and electron microscopy imaging techniques, the protein in live cells or tissue can be studied at higher resolution at the ultrastructural level, making correlative light and electron microscopy a very powerful tool $[1,2]$.

Project variety is the nature of the core facility. Experimental design of complex procedures, such as CLEM for each individual project by utilizing current available instruments and reagents, is a challenge. We have successfully carried on multiple projects by using different CLEM strategies [1], such as: neighboring sections, hybrid labeling, correlative overlay and Tokuyasu cryosections. To study cortical inhibitory neuron maturation and synaptic development, we use hybrid labeling strategy to double immunolabel GFP and Somatostatin (SST, a growth hormone-inhibiting hormone) of brain vibrotome sections of the Lhx6-eGFP transgenic mouse, to detect: (1) GFP positive cells under fluorescent microscope following correlated ultrastructural immunolabeling using Nanogold as marker; (2) SST positive cells using HRP/DAB as marker (Fig. 1). Double positive cells (Fig. 1) and single positive cells (data not shown) were clearly distinguished by silver enhanced gold particles and DAB darkened cells at ultrastructure level. To study melanoma invasion, we used the frozen section correlative overlay strategy to localize the melanocyte migration in the skin of Braf and P10 knock out mouse (Fig. 2). The gridded bottom dish was adopted for several projects to study centrosome mutation [3] and HIV infection (not published) using correlative overlay strategy; and Tokuyasu cryosections combined with super resolution microscopy were used to study the protein localization in intercalated disc of mouse heart [4]. 


\section{References:}

[1] K. Cortese et al, The Journal of Histochemistry and Cytochemistry 57, (2009), 1103-12.

[2] B. N. Giepmans, Histochemstry and Cell Biology 130, (2008), 211-217.

[3] J. Li et al, Nature 495, (2013), 255-259.

[4] E. Agullo-Pascual et al, Cardiovascular Research 104 (2), (2014), 371-381.
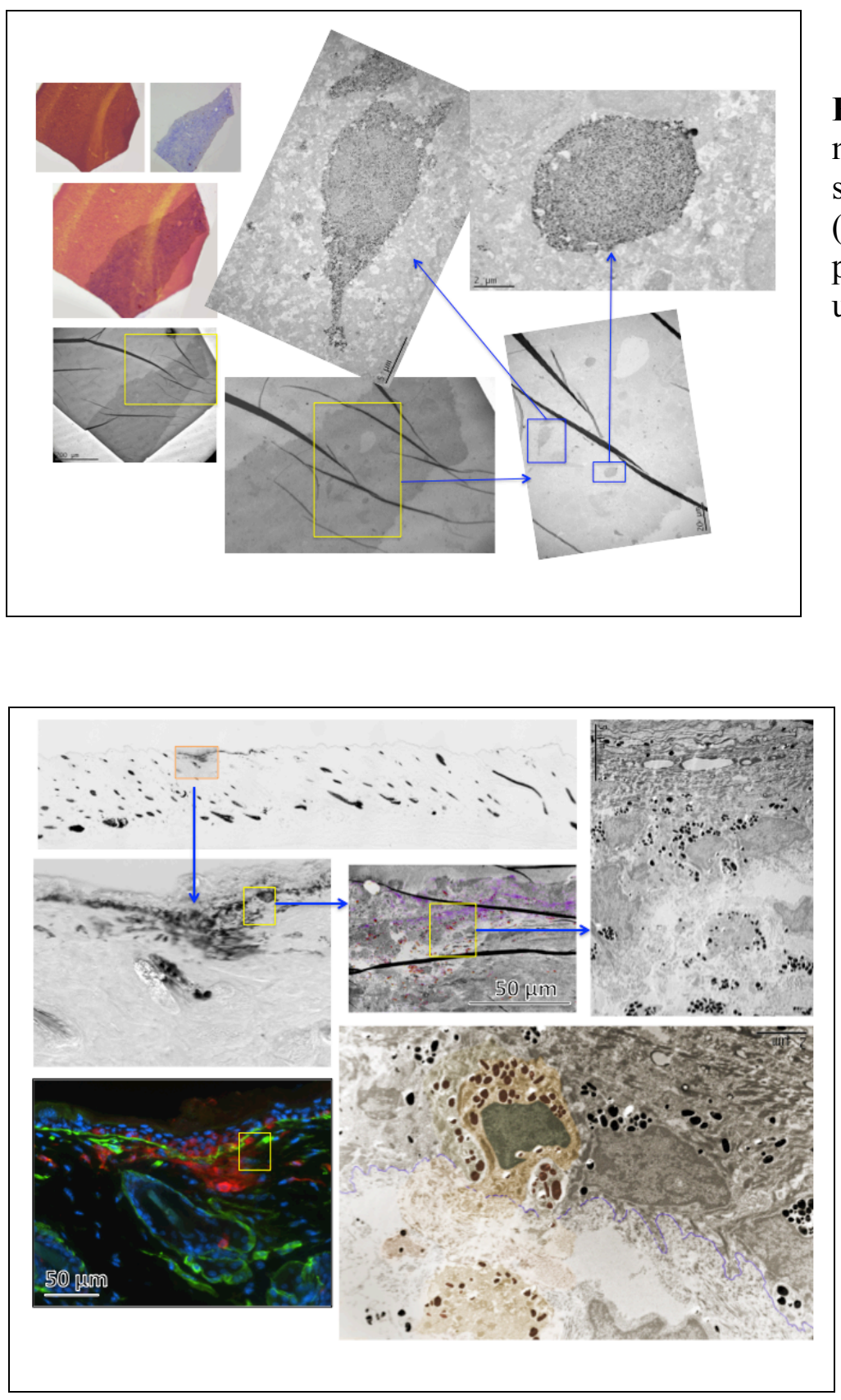

Figure 1. Correlative light and electron microscopy study using brain vibrotome section to double immunolocalize GFP (Nanogold) and SST (HRP/DAB). Double positive cells were shown clearly at ultrastructural level.
Figure 2. Melanoma cells invasion in mouse skin was identified by using frozen section correlative overlay strategy. 